\title{
Zur Biosynthese von 2.3-Dihydroxybenzoesäure in Submerskulturen von Claviceps paspali Stevens et Hall
}

\author{
Detlef Gröger und Dieter Erge \\ Institut für Biochemie der Pflanzen der Deutschen Akademie der Wissenschaften zu Berlin, Halle (Saale) \\ und Heinz-Günter Floss \\ Organisch-Chemisches Institut der Technischen Hochschule München \\ (Z. Naturforschg. 20 b, 856-858 [1965] ; eingegangen am 9. Juni 1965) \\ Claviceps paspali vermag Tryptophan über Anthranilsäure zu 2.3-Dihydroxybenzoesäure (DOB) \\ abzubauen. Kynurenin ist wahrscheinlich eine Zwischenstufe in dieser Reaktionsfolge. Die Um- \\ wandlung von Anthranilsäure in DOB erfolgt vermutlich in einem Schritt, da markierte Salicyl- \\ säure und 3-Hydroxyanthranilsäure nicht in DOB eingebaut wurden.
}

2.3-Dihydroxybenzoesäure $(=\mathrm{DOB})$ ist in höheren Pflanzen sowie in Mikroorganismen nachgewiesen worden. Sie kommt besonders in einigen Gattungen der Ericaceae, Asclepiadaceae und Apocynaceae $^{1}$ sowie der Saxifragaceae ${ }^{2}$ vor. Iто und Neilands ${ }^{3}$ konnten aus Bacillus subtilis-Kulturen 2.3-Dihydroxybenzoylglycin isolieren, wenn das Fermentationsmedium wenig Eisen enthielt. Ebenfalls in eisenarmem Medium bildet Streptomyces griseus DOB bei gleichzeitiger starker Hemmung der Streptomycinsynthese ${ }^{4,5}$, Terui et al. ${ }^{6}$ fanden DOB in Kulturen von Aspergillus niger und ARCAMONE et. $\mathrm{al}^{7}$ konnten diese Phenolcarbonsäure aus Submerskulturen von $\mathrm{Cl}$. paspali in einer Ausbeute von $200-300 \mathrm{mg} / l$ Nährlösung kristallin gewinnen.

Zur Biosynthese der DOB liegen mehrere Untersuchungen vor. Iвrahim und Towers ${ }^{8}$ konnten zeigen, daß Salicylsäure zur DOB oxydiert wird. Später wies Івванім ${ }^{9}$ nach, daß auch Benzoesäure, Phenylalanin und Zimtsäure als Vorstufen für DOB in Blättern von Primula und Gaultheria dienen können. Zu gleichen Ergebnissen kamen EL-BasyounI et al. ${ }^{10}$. Kindu und BiLleK ${ }^{11}$ wiesen nach, daß Blätter von Hydrangea macrophylla und Astilbe chinensis Benzoesäure zur DOB hydroxylieren.

Andere Bildungswege existieren in Mikroorganismen. So fanden Pittard et al. ${ }^{12}$ Akkumulation von DOB durch Tryptophan- und Tyrosin-auxotrophe Mutanten von Aerobacter aerogenes in einem Mine-

1 R. K. Івrahim, Naturwissenschaften 50, 734 [1963].

2 G. Billek u. H. Kinde, Mh. Chem. 93, 85 [1962].

3 T. Ito u. J.B. Neilands, J. Amer. Chem. Soc. 80, 4645 [1958].

4 V. Musilek u. R. Nomi, Abstracts Intern. Congr. Microbiol. (Montreal), p. 66 [1962].

5 J. R. Dyer, H. Heding u. C. P. Schaffer, J. org. Chemistry 29, 2802 [1964].

${ }^{6}$ G. Terui, T. Enatzu u. S. Tabota, J. Ferment. Technol. 39, 224 [1961].

7 F. Arcamone, E. B. Chain, A. Ferretti u. P. Pennella, Nature [London] 192, 552 [1961].
ralsalz-Glucose-Medium. Aus den Versuchen dieser Autoren geht hervor, daß die DOB hier wahrscheinlich aus noch nicht aromatischen Zwischenprodukten des Shikimisäureweges entsteht. Durch Replacementversuche konnten Arcamone et al. ${ }^{7}$ zeigen, daß in Cl. paspali DOB wahrscheinlich ein Abbauprodukt des Tryptophans ist. Wir ${ }^{13}$ konnten diesen Befund durch Verfütterung von radioaktiv markiertem Tryptophan an Submerskulturen von $\mathrm{Cl}$. paspali endgültig beweisen. Da es sich hierbei um einen neuen Abbauweg der Aminosäure Tryptophan handelt, schien es wünschenswert, den Mechanismus dieser Reaktion näher zu untersuchen. Ausgehend vom Tryptophan sind mehrere Bildungswege für die DOB möglich:

1. Tryptophan wird über mehrere Stufen zur Salicylsäure abgebaut (s. auch Proctor ${ }^{14}$ ), die dann zur DOB hydroxyliert wird.

2. Bildung von 3-Hydroxyanthranilsäure via Kynurenin $\rightarrow 3-\mathrm{OH}-\mathrm{Kynurenin}$, die durch oxydative Desaminierung in DOB übergeht.

3. Abbau von Tryptophan zur Anthranilsäure, die dann hydroxyliert und desaminiert wird.

\section{Material und Methoden}

Markierte Verbindungen: Anthranilsäure-[carboxyl${ }^{14} \mathrm{C}$ ], wurde nach der Vorschrift von Munsche und ScHÜтte ${ }^{15}$ hergestellt. L-Tryptophan-[T], 3-Hydroxyanthranilsäure-[T] und DL-Kynurenin-[T] wurden durch Austausch mit $\mathrm{HO}^{3} \mathrm{H}$ und konz. HCl bei $110^{\circ}$

8 R. K. Iвrahim u. G. H. N. Towers, Nature [London] 184, 1803 [1959].

9 R. K. Івrahim, Flora [Jena] 154, 481 [1964].

10 S. Z. El-Basyouni, D. Chen, R. K. Ibrahim, A. C. Neish u. G. H. N. Towers, Phytochemistry 3, 485 [1964].

11 H. Kindl u. G. Billek, Mh. Chem. 95, 1044 [1964].

12 A. J. Pittard, F. Gibson u. C. H. Doy, Biochim. biophysica Acta [Amsterdam] 57, 290 [1962].

13 V. E. Tyler Jr., K. Mothes, D. Gröger u. H.-G. Floss, Tetrahedron Letters 1964, 593.

14) M. H. Proctor, Nature [London] 181, 1345 [1958]. 
und anschließenden Rücktausch des labilen Tritiums erhalten. Salicylsäure-[carboxyl- ${ }^{14} \mathrm{C}$ ] wurde vom Radiochemical Center, Amersham, bezogen.

Kulturtechnik: Für die Versuche wurde der von GRöger und Tyler ${ }^{\mathbf{1 6}}$ isolierte $C l$. paspali-Stamm Li 189 verwendet: Das Mycel eines 14 Tage alten Agarröhrchens wurde abgeschabt und in 50-ml-Rundkolben, die $100 \mathrm{ml}$ einer Nährlösung (mit corn steep solids „Staley“) enthielten, übertragen. Nach 5-tägigem Schütteln bei $24^{\circ}$ waren die Kolben zugewachsen. Diese Vorkulturen wurden abgesaugt, mit sterilem Wasser gewaschen und jeweils $1 / 4$ einer Vorkultur in einen Rundkolben mit $100 \mathrm{ml}$ Fermentationslösung transferiert. Fermentationsmedium [g]: Ammoniumsuccinat 30,0 ; Mannit 30,$0 ; \mathrm{KH}_{2} \mathrm{PO}_{4} 1,0 ; \mathrm{MgSO}_{4} \cdot 7 \mathrm{H}_{2} \mathrm{O} 0,3$; 1.2-Propandiol $30 \mathrm{ml}$; Aqua dest ad $1000,0 \mathrm{ml}$. Der $p_{\mathrm{H}}$-Wert wurde mit 10 -proz. $\mathrm{HCl}$ auf $5,1-5,2$ eingestellt. Die Sterilisation erfolgte im Autoclaven. Die zu applizierenden Verbindungen wurden in einigen $\mathrm{ml}$ der Nährlösung gelöst, durch ein Bakterienfilter gegeben und vor der Beimpfung zum Fermentationsmedium hinzugegeben. Nach 4-tägigem Schütteln bei $24{ }^{\circ} \mathrm{C}$ wurden die Versuche abgebrochen und die Nährlösung vom Mycel getrennt.

Aufarbeitung: Der Gehalt an DOB in der Nährlösung wurde kolorimetrisch nach GRöGER und ERGE ${ }^{17}$ bestimmt. Aus der Nährlösung wurde die DOB nach dem von uns angegebenen Verfahren ${ }^{\mathbf{1 3}}$ durch Chromatographie an Ionenaustauscher-Säulen Dowex $1 \times 10$ isoliert. Bei Anthranilsäure-Applikation muß auf die restlose Entfernung dieser Säure durch Elution mit 1- $n$. Ameisensäure besonders geachtet werden. Zur völligen Abtrennung der Salicylsäure wurde die erhaltene DOB mehrfach im System Benzol : Eisessig : Wasser ${ }^{2}$ (4 : $2: 1)$ auf Schleicher \& Schüll-Papier $2043 \mathrm{bm}$ absteigend chromatographiert. Die isolierten DOB-Präparate wurden durch Schmelzpunkt und IR-Spektrum charakterisiert und jeweils im System Propanol : Wasser $(3: 1)$ aufsteigend chromatographisch untersucht. Sie wurden ferner mit Trägermaterial verdünnt und bis zur konstanten spezifischen Aktivität aus Wasser bzw. Methanol umkristallisiert. Zur Decarboxylierung wurde

die DOB mit Kupferpulver im Bombenrohr mit „,break tip" $1^{1 / 2}$ Stdn. auf $220^{\circ}$ erhitzt. Das $\mathrm{CO}_{2}$ wurde in ein Proportionalzählrohr überführt.

Alle Radioaktivitäts-Analysen erfolgten durch Gasphasenmessung nach der Methode von Simon ${ }^{18}$.

\section{Ergebnisse und Diskussion}

Die Ergebnisse sind in den Tabn. 1 und 2 zusammengefaßt. Auf Grund der hohen Einbaurate kann man annehmen, daß Anthranilsäure eine unmittelbare Vorstufe der 2.3-Dihydroxybenzoesäure bei $\mathrm{Cl}$. paspali ist. Ein Teil der nach Fütterung von Anthranilsäure-[carboxyl $-{ }^{14} \mathrm{C}$ ] erhaltenen DOB wurde mit nichtradioaktiver $\mathrm{DOB}$ verdünnt und decarboxyliert. Die für diesen Versuch eingesetzte DOB enthielt $1945 \mathrm{dpm} / \mu \mathrm{Mol}$ und das erhaltene $\mathrm{CO}_{2}$ besaß eine spezifische Aktivität von $1930 \mathrm{dpm} /$ $\mu \mathrm{Mol}$. Damit ist bewiesen, daß ein direkter Übergang Anthranilsäure $\rightarrow$ DOB erfolgt sein muß und daß eine Decarboxylierung der Anthranilsäure und ein unspezifischer Einbau von $\mathrm{CO}_{2}$ in DOB ausgeschlossen werden kann. Hydroxylierungen der Anthranilsäure, bei denen die Carboxylgruppe erhalten bleibt, sind in der Natur bisher nur selten beobachtet worden. Über den Mechanismus dieser Reaktion können wir noch keine Aussagen machen. Es wäre denkbar, daß die Umwandlung Anthranilsäure $\rightarrow$ DOB analog der Bildung von Catechol aus Anthranilsäure in Pseudomonas fluorescens abläuft, deren Mechanismus kürzlich von Kовауаsнi et al. ${ }^{19}$ aufgeklärt werden konnte. Untersuchungen mit $\mathrm{H}_{2}{ }^{18} \mathrm{O}$ und ${ }^{18} \mathrm{O}_{2}$ sind im Gange. Interessant sind in diesem Zusammenhang unsere früheren Befunde ${ }^{17}$. Nach Verfütterung von Tryptophan an $\mathrm{Cl}$. paspali (kultiviert in einem Vollmedium) ließen sich neben DOB

\begin{tabular}{|c|c|c|c|c|c|}
\hline appliziert & $\begin{array}{l}\text { DOB } \\
\text { im Medium } \\
{[\mathrm{mg}]}\end{array}$ & $\begin{array}{c}\text { Gesamtakt. } \\
\text { der DOB } \\
{[\mathrm{Ipm}]}\end{array}$ & $\begin{array}{l}\text { Einbaurate } \\
{[\%]}\end{array}$ & $\begin{array}{c}\text { spez. Akt. } \\
\text { der DOB } \\
{[\mathrm{dpm} / \mathrm{mMol}]}\end{array}$ & $\begin{array}{c}\text { spez. } \\
\text { Einbaurate } \\
{[\%]}\end{array}$ \\
\hline $\begin{array}{l}\left.5,2 \mathrm{mg} \text { Anthranilsäure-[carboxyl- }{ }^{14} \mathrm{C}\right] \\
4,0 \cdot 10^{7} \mathrm{dpm}\end{array}$ & 20,2 & $2,0 \cdot 10^{7}$ & 50 & $1,54 \cdot 10^{8}$ & 14,5 \\
\hline $\begin{array}{l}5,5 \mathrm{mg} 3-\mathrm{Hydroxy} \text {-anthranilsäure-(т) } \\
1,33 \cdot 10^{7} \mathrm{dpm}\end{array}$ & 12 & $1,44 \cdot 10^{5}$ & 1,05 & $1,85 \cdot 10^{6}$ & 0,5 \\
\hline $\begin{array}{l}0,65 \mathrm{mg} \text { D L-Kynurenin-(T) } \\
1,06 \cdot 10^{7} \mathrm{dpm}\end{array}$ & 23 & $3,75 \cdot 10^{5}$ & 3,52 & $2.52 \cdot 10^{6}$ & 0,075 \\
\hline $\begin{array}{l}0,5 \mathrm{mg} \text { Salicylsäure-[carboxyl-14C] } \\
3,46 \cdot 10^{7} \mathrm{dpm}\end{array}$ & 22,6 & $8,35 \cdot 10^{4}$ & 0,24 & $5,7 \cdot 10^{5}$ & 0,006 \\
\hline
\end{tabular}

Tab. 1. Der Einbau von Anthranilsäure- $\left[{ }^{14} \mathrm{COOH}\right]$ (spez. Akt. 1,06·10 $\mathrm{dpm} / \mathrm{mMol}$ ) ; 3-Hydroxyanthranilsäure-(T) (spez. Akt. $3,7 \cdot 10^{8} \mathrm{Ipm} / \mathrm{mMol}$ ); DL-Kynurenin als Sulfat appliziert $\left(3,4 \cdot 10^{9} \mathrm{dpm} / \mathrm{mMol}\right.$ ); Salicxylsäure- $\left[{ }^{14} \mathrm{COOH}\right]$ (spez. Akt. $9,6 \cdot 10^{9} \mathrm{Ipm} / \mathrm{mMol}$ ) in 2.3-Dihydroxybenzoesäure (DOB).

15 D. Munsche U. H. R. Schütte, Z. Chem. 3, 230 [1963].

16 D. Gröger u. V. E. Tyler Jr., Lloydia 26, 174 [1963].
17 D. Gröger u. D. ERge, Pharmazie 19, 775 [1964].

18 H. Simon, H. Daniel u. J. F. Klebe, Angew. Chem. 71, 303 [1959]. 


\begin{tabular}{|c|c|c|c|c|c|}
\hline Versuchs-Nr. & appliziert & $\begin{array}{r}2.3-\mathrm{DOB} \\
{[\mathrm{mg}]}\end{array}$ & $\begin{array}{c}\text { Einbaurate } \\
{[\%]}\end{array}$ & $\begin{array}{c}\text { spez. Akt. d. } \\
2.3-\mathrm{DOB} \\
{[\mathrm{dpm} / \mathrm{mMol}]}\end{array}$ & $\begin{array}{c}\text { spezifische } \\
\text { Einbaurate } \\
{[\%]}\end{array}$ \\
\hline $20 \mathrm{~B} 1$ & 6,0 mg Tryptophan-[T] $2,45 \cdot 10^{8} \mathrm{dpm}$ & 11.7 & 20 & $6,42 \cdot 10^{8}$ & 7,6 \\
\hline 20 B 2 & $\begin{array}{l}6 \mathrm{mg} \text { Tryptophan-[T] } 2,45 \cdot 10^{8} \mathrm{dpm}+ \\
6,1 \mathrm{mg} \text { L-Kynurenin }\end{array}$ & 17 & 22 & $5,04 \cdot 10^{8}$ & 6 \\
\hline $20 \mathrm{~B} 3$ & $\begin{array}{l}6 \text { mg Tryptophan-[T] } 2,45 \cdot 10^{8} \mathrm{dpm}+ \\
11,4 \mathrm{mg} \text { L-Kynurenin }\end{array}$ & 18,5 & 19,5 & $3,83 \cdot 10^{8}$ & 4,5 \\
\hline $20 \mathrm{~B} 4$ & $\begin{array}{l}5,64 \mathrm{mg} \text { Tryptophan-[T] } 2,3 \cdot 10^{8} \mathrm{dpm}+ \\
9,7 \mathrm{mg} \text { D L-Kynurenin }\end{array}$ & 18,5 & 17 & $3,26 \cdot 10^{8}$ & 3,85 \\
\hline
\end{tabular}

Tab. 2. Einbau von L-Tryptophan-indol-T (spez. Akt. $3,85 \mathrm{mC} / \mathrm{mMol}$ ) in 2.3-Dioxybenzoesäure bei gleichzeitiger Verfütterung von Kynurenin in $\mathrm{Cl}$. paspali-Submerskulturen.

nur Spuren von Anthranilsäure nachweisen. Wurde der Pilz dagegen in einem stark verdünnten nährstoffarmen Medium kultiviert, kam es zu einer Anreicherung von Anthranilsäure und die Bildung von DOB trat zurück. Die Frage ist noch offen, ob die DOB nur aus der beim Tryptophanabbau entstehenden Anthranilsäure hervorgeht oder auch direkt aus Anthranilsäure, die von der Shikimisäure stammt, also ohne den Umweg über das Tryptophan.

Die 3-Hydroxyanthranilsäure ist eine wesentlich schlechtere Vorstufe für die DOB als die Anthranilsäure, wie aus Tab. 1 hervorgeht. Die Hydroxy. lierung dieser Säure scheint höchstens als Nebenweg für die DOB-Synthese in Frage zu kommen. Andererseits ist zu bedenken, daß 3-OH-Anthranilsäure eine verhältnismäßig labile Substanz ist, die leicht oxydiert wird. Ferner ist über die Permeabilität dieser Säure wenig bekannt und es ist denkbar, daß nur ein geringer Teil der Vorstufe unzersetzt an den Syntheseort gelangt. Überraschend war der Befund nach Kynureninfütterung. Entsteht die DOB ausgehend von Tryptophan via Kynurenin $\rightarrow$ Anthranilsäure, so sollte man annehmen, daß Kynurenin besser verwertet wird als Tryptophan. Dies ist jedoch bei diesen Versuchen nicht der Fall. Da wir aber Tritium-markiertes Kynurenin eingesetzt haben, das wahrscheinlich einen großen Teil der Radioaktivität in der Methylengruppe der Seitenkette enthält, ist dieser negative Befund nicht unbedingt beweisend. Bei der Umwandlung von Kynurenin in Anthranilsäure geht die Radioaktivität der Seiten-

19 S. Kobayashi, S. Kuno, N. Itada, O. Hayaishi, S. Kozuka u. S. OAE, Biochem. biophysic. Res. Commun. 16, 556 [1964].

20 W. H. Matchett u. J. A. DeMoss, Biochim. biophysica Acta [Amsterdam] 71, 632 [1963].

21 F. Arcamone, E. B. Chain, A. Ferretti, A. Minghetti, P. Pennella u. A. Tonolo, Biochim. biophysica Acta [Amsterdam] 57, 174 [1962].

22 F. Baum, Z. allg. Mikrobiol. 4, 185 [1964]. kette natürlich verloren. Zur weiteren Untersuchung haben wir einen Konkurrenzversuch durchgeführt und markiertes Tryptophan mit inaktivem Kynurenin gleichzeitig verfüttert. Die Ergebnisse sind in Tab. 2 zusammengestellt. Aus den spez. Einbauraten geht hervor, daß Kynurenin den Einbau von Tryptophan in die DOB hemmt. Die gleichzeitige Applikation von Kynurenin führt außerdem zu einer Erhöhung der Ausbeute an DOB. Dies sind indirekte Beweise, daß Kynurenin als eine Vorstufe der DOB anzusehen ist, die in der Synthesekette hinter dem Tryptophan liegt. Offen bleibt die Frage, ob es nicht noch einen anderen Weg vom Tryptophan zur Anthranilsäure gibt, der nicht über Kynurenin führt. Es käme beispielsweise der in Neurospora gefundene Parallelweg über Formylanthranilsäure in Frage ${ }^{20}$. Diese Verbindung ist in Replacement-Kulturen von $\mathrm{Cl}$. paspali nachgewiesen worden ${ }^{21}$.

Nach Applikation von Salicylsäure- $\left[{ }^{14} \mathrm{COOH}\right]$ konnte nur außerordentlich schwach markierte DOB isoliert werden (Tab. 1). Die spez. Einbaurate betrug 0,006 Prozent. Offenbar ist unser Pilzstamm, im Gegensatz zu höheren Pflanzen, nicht in der Lage, Salicylsäure direkt zu hydroxylieren. Bis heute sind wohl noch keine Beispiele dafür bekannt, daß Mikroorganismen Salicylsäure in DOB überführen können (s. a. BAUM ${ }^{22}$ ).

Zusammenfassend läßt sich sagen, daß unser $\mathrm{Cl}$. paspali-Stamm ausgehend von Tryptophan (via Kynurenin?) über Anthranilsäure die DOB synthetisiert. Der Weg über Salicylsäure kann wohl ausgeschlossen werden. Die Nichtverwertung der 3-OH-Anthranilsäure zur DOB-Synthese muß noch weiter erhärtet werden. Neben den bisher bekannten Biosynthesewegen, die zu Phenolcarbonsäuren führen, ist hier ein neuer aufgezeigt; denn zumindest eine der bekannten hydroxylierten Benzoesäuren kann über Anthranilsäure entstehen. 